\title{
Polyaniline and manganese oxide decorated on carbon nanofibers as a superior electrode material for supercapacitor
}

\begin{abstract}
A novel ternary composite of carbon nanofibers/polyaniline-manganese oxide (CNFs/PANI$\mathrm{MnO} 2$ ) was synthesized via electrospinning, carbonization followed by electrodeposition of PANI-MnO2. The CNFs/PANI-MnO2 composite exhibited excellent specific capacitance of 937.66 F/g at a scan rate of $5 \mathrm{mV} / \mathrm{s}$ and good cyclic stability with capacitance retention of $97.6 \%$ after 5000 consecutive cycles. The composite also exhibited superior performance with a specific energy of $66.12 \mathrm{Wh} / \mathrm{kg}$ at a specific power of $470.81 \mathrm{~W} / \mathrm{kg}$ with low charge transfer resistance, Rct (1.81 $\Omega$ ) and equivalent series resistance (32.18 $\Omega$ ) indicating high electronic conductivity. Three symmetrical CNFs/PANI-MnO2 composites assembled in series using coin cells have successfully lighted up a red-light emitting diode (LED), proving its outstanding supercapacitive performance as an excellent electrode material for supercapacitors.
\end{abstract}

Keyword: Supercapacitor; Manganese oxide; Carbon nanofibers; Polyaniline; Specific capacitance; Coin cell 